\title{
Adenosine kinase inhibition promotes survival of fetal adenosine deaminase-deficient thymocytes by blocking dATP accumulation
}

\author{
C. Justin Van De Wiele, ${ }^{1,2}$ James G. Vaughn, ${ }^{1}$ Michael R. Blackburn, ${ }^{3}$ Catherine A. Ledent, ${ }^{4}$ \\ Marlene Jacobson, ${ }^{5}$ Hong Jiang, ${ }^{1}$ and Linda F. Thompson ${ }^{1,2}$
}

\author{
${ }^{1}$ Immunobiology and Cancer Program, Oklahoma Medical Research Foundation, Oklahoma City, Oklahoma, USA \\ ${ }^{2}$ Department of Microbiology and Immunology, University of Oklahoma, Oklahoma City, Oklahoma, USA \\ ${ }^{3}$ Department of Biochemistry, University of Texas/Houston Medical School, Houston, Texas, USA \\ ${ }^{4}$ Université Libre de Bruxelles, Brussels, Belgium \\ ${ }^{5}$ Merck Research Laboratories, West Point, Pennsylvania, USA
}

\begin{abstract}
Thymocyte development past the $\mathrm{CD}^{-} \mathrm{CD}^{-}$stage is markedly inhibited in adenosine deaminase-deficient (ADA-deficient) murine fetal thymic organ cultures (FTOCs) due to the accumulation of ADA substrates derived from thymocytes failing developmental checkpoints. Such cultures can be rescued by overexpression of Bcl-2, suggesting that apoptosis is an important component of the mechanism by which ADA deficiency impairs thymocyte development. Consistent with this conclusion, ADA-deficient FTOCs were partially rescued by a rearranged T cell receptor $\beta$ transgene that permits virtually all thymocytes to pass the $\beta$-selection checkpoint. ADA-deficient cultures were also rescued by the adenosine kinase inhibitor $5^{\prime}$-amino- $5^{\prime}$-deoxyadenosine $\left(5^{\prime} \mathrm{A} 5^{\prime} \mathrm{dAdo}\right)$, indicating that the metabolite responsible for the inhibition of thymocyte development is not adenosine or deoxyadenosine, but a phosphorylated derivative of an ADA substrate. Correction of ADA-deficient FTOCs by $5^{\prime} \mathrm{A} 5^{\prime} \mathrm{dAdo}$ correlated with reduced accumulation of dATP, implicating this compound as the toxic metabolite. In ADA-inhibited FTOCs rescued with a $\mathrm{Bcl}-2$ transgene, however, dATP levels were superelevated, suggesting that cells failing positive and negative selection continued to contribute to the accumulation of ADA substrates. Our data are consistent with dATP-induced mitochondrial cytochrome $c$ release followed by apoptosis as the mechanism by which ADA deficiency leads to reduced thymic $\mathrm{T}$ cell production.
\end{abstract}

J. Clin. Invest. 110:395-402 (2002). doi:10.1172/JCI200215683

\section{Introduction}

Adenosine deaminase (ADA) catalyzes the irreversible deamination of adenosine and deoxyadenosine to inosine and deoxyinosine, respectively. Mutations in the ADA gene that result in loss of enzyme activity cause severe combined immunodeficiency (1). Biochemical aberra-

Received for publication April 12, 2002, and accepted in revised form June 11, 2002.

Address correspondence to: Linda F. Thompson, Oklahoma Medical Research Foundation, 825 NE 13th Street, Oklahoma City, Oklahoma 73104, USA. Phone: (405) 271-7235;

Fax: (405) 271-8568; E-mail: Linda-Thompson@omrf.ouhsc.edu. C. Justin Van De Wiele's present address is: Department of Surgery, College of Medicine, University of Oklahoma, Tulsa, Oklahoma, USA.

Hong Jiang's present address is: Curatek Pharmaceuticals, Elk Grove Village, Illinois, USA.

Conflict of interest: No conflict of interest has been declared. Nonstandard abbreviations used: adenosine deaminase (ADA); fetal thymic organ culture (FTOC); S-adenosylhomocysteine (SAH); T cell receptor $\beta$ transgene (TCR $\beta$ ); carbobenzoxy-Val-AlaAsp-fluoromethyl ketone (z-VADfmk); apoptotic proteaseactivating factor-1 (Apaf-1); adenosine receptor $2 \mathrm{a}(\mathrm{A} 2 \mathrm{aR})$; $2^{\prime}$-deoxycoformycin (17) (dCF); $5^{\prime}$-N-ethylcarboxamidoadenosine (NECA); xanthine amine congener (XAC); $5^{\prime}$-amino-5'-

deoxyadenosine (5'A5'dAdo); ethidium bromide (EtBr); double positive (DP); S-adenosylmethionine (SAM); phycoerythrin (PE); propidium iodide (PI). tions due to ADA deficiency have been delineated over the past 30 years, but it is still unclear why loss of this enzyme activity exhibits such profound effects on the immune system (reviewed in ref. 2). Adenosine and deoxyadenosine, the substrates of ADA, are generated in the microenvironment of emerging thymocytes through normal mechanisms of lymphocyte selection. Thymocytes failing developmental checkpoints die and are degraded by thymic macrophages (3) generating adenosine and deoxyadenosine $(4,5)$. In a normal thymus, ADA catabolizes these metabolites, but in ADA deficiency they accumulate $(6,7)$ and exert lymphotoxic effects either directly (2) or after conversion to phosphorylated derivatives such as AMP and dATP (2, 8-11). In an environment where up to $95 \%$ of the cells undergo programmed cell death, it is easy to visualize the potential of a cell to accumulate toxic levels of purine metabolites.

ADA-deficient murine fetal thymic organ culture (FTOC) is an excellent model of the human disease (12) because it exhibits many biochemical features of ADA-deficient patients, including ADA substrate and dATP accumulation as well as S-adenosylhomocysteine (SAH) hydrolase inhibition. Furthermore, the yield of thymocytes from ADA-deficient cultures is $85-95 \%$ less than in control cultures, with thymocyte development becoming progressively more impaired 
past the $\mathrm{CD} 4{ }^{-} \mathrm{CD} 8^{-} \mathrm{CD} 25^{+} \mathrm{CD} 44^{-}$stage, the point where $T$ cell receptor $\beta(T C R \beta) \mathrm{V} \rightarrow \mathrm{DJ}$ gene rearrangements occur. This is presumably because of the accumulation of ADA substrates derived from thymocytes that fail to rearrange an in-frame TCR $\beta$ chain. This conclusion is supported by rescue of ADA-inhibited FTOCs by the pan-caspase inhibitor carbobenzoxyVal-Ala-Asp-fluoromethyl ketone (z-VADfmk). Inhibition of caspase activity prevents the accumulation of dATP, most likely by preventing the apoptotic death of thymocytes failing $\beta$ selection. ADA-deficient FTOCs are also rescued by a $B c l-2$ transgene or deletion of apoptotic protease-activating factor-1 (Apaf-1), providing further evidence of the involvement of apoptosis in the inhibition of lymphocyte development observed in our cultures. However, whether these latter cultures were rescued by prevention of substrate accumulation or by direct intervention in the mechanism of toxicity mediated by ADA substrates was not determined.

Adenosine, deoxyadenosine, AMP, and dATP have all been implicated in causing the lymphopenia observed in ADA deficiency (2). Aberrant adenosine receptor engagement can induce thymocyte apoptosis in vitro. Inhibition of pyrimidine synthesis by AMP can starve developing cells of molecules required for both RNA and DNA production. Inhibition of SAH hydrolase by both adenosine and deoxyadenosine can result in feedback inhibition of methylation reactions required for cell viability. Inhibition of ribonucleotide reductase by dATP can prevent production of DNA precursors and thus inhibit cell proliferation. Phosphorylation events coupled with dATP-mediated stimulation of adenine ribonucleotide catabolism can deplete cellular ATP. Finally, dATP can induce mitochondrial cytochrome $c$ release and subsequent apoptosis (11). We show here that normalization of dATP levels by inhibition of adenosine kinase abrogates the effects of ADA deficiency in FTOC, suggesting that dATP is the toxic metabolite. Furthermore, since our cultures can also be rescued by a $B c l-2$ transgene, our data are consistent with dATP-induced cytochrome $c$ release from mitochondria followed by initiation of the apoptotic cascade as the mechanism of toxicity.

\section{Methods}

Mice. C57BL/6 mice (Taconic Farms, Germantown, New York, USA or The Jackson Laboratory, Bar Harbor, Maine, USA) and transgenic mice expressing $B c l-2$ under the control of the lck proximal promoter (The Jackson Laboratory) (13) were purchased from the indicated sources. Transgenic mice expressing a rearranged $\mathrm{OVA}_{257-264}$-specific TCR $\beta$ chain (14) were obtained from Francis Carbone (Monash University, Melbourne, Australia). ADA-deficient (7), adenosine receptor $2 \mathrm{a}$-deficient (A2aR-deficient) (15), and A3R-deficient mice (16) have been described previously. The Ada gene-targeted allele has been designated $m 1$. Because $A d a^{m 1 / m 1}$ mice are not viable, $A d a^{m 1 /+}$ mice were bred to each other to obtain Ada ${ }^{m 1 / m 1}$ fetal thymuses for FTOCs. Ada ${ }^{m 1 /+}$ and
$A 3 R^{-/-}$mice were crossed to produce $A d a^{m 1 /+} A 3 R^{-/-}$mice. These mice were bred to each other to obtain Ada $a^{m 1 / m 1} A 3 R^{-1-}$ fetal thymuses for FTOC. All mice were bred and maintained in our animal facility under specific pathogen-free conditions (Laboratory Animal Resource Center, Oklahoma City, Oklahoma, USA) in accordance with procedures outlined in "Guide to Care and Use of Laboratory Animals" (National Research Council, Washington, D.C., USA).

Drugs and reagents. The specific ADA inhibitor, 2'-deoxycoformycin (17) (dCF), was obtained from Parke Davis (Ann Arbor, Michigan, USA) or SuperGen (Dublin, California, USA). The general adenosine receptor agonist $5^{\prime}$ - $N$-ethylcarboxamidoadenosine (NECA), the general adenosine receptor antagonist 8-[4-[[[[(2aminoethyl)amino]carbonyl]methyl]oxy]phenyl]-1,3dipropylxanthine (xanthine amine congener, or XAC), and the adenosine kinase inhibitor, $5^{\prime}$-amino- $5^{\prime}$ deoxyadenosine (18) (5'A5'dAdo) were obtained from Sigma-Aldrich (St. Louis, Missouri, USA).

FTOC. Thymuses were removed from fetuses derived from timed pregnant mice on day 14 or 15 of gestation and cultured in FTOC as described previously (12). FTOCs with ADA-deficient lobes were performed in serum-free medium, HyQ-CCM1 (HyClone Laboratories, Logan, Utah, USA), supplemented with glutamine and antibiotics. In experiments with C57BL/ 6 fetuses, thymuses were separated into individual lobes, randomized, and cultured in the presence or absence of $5 \mu \mathrm{M} \mathrm{dCF}$ and/or test reagents. In FTOCs with transgenic or genetargeted mice, one lobe from each thymus was cultured under control conditions and the other under experimental conditions. At harvest, lobes of the pertinent genotypes were pooled and pushed through $70-\mu \mathrm{m}$ nylon screens to make single cell suspensions. Cells were then counted and characterized for cell surface phenotype.

Genotyping. ADA-deficient thymuses derived from breeding either $A d a^{m 1 /+}$ or $A d a^{m 1 /+} A 3 R^{-/-}$mice were identified by ADA enzyme assays (19) on the corresponding fetal livers. TCR $\beta$ chain and $B c l-2$ transgenic fetuses were identified by PCR on DNA extracted from fetal livers by digestion overnight at $55^{\circ} \mathrm{C}$ in proteinase $\mathrm{K}$, followed by phenol/chloroform extraction and ethanol precipitation. For PCR, the following conditions were used in a $20-\mu \mathrm{l}$ reaction: $2.5 \mathrm{mM}$ $\mathrm{MgCl}_{2}, 200 \mu \mathrm{M}$ dNTPs, $1 \mu \mathrm{M}$ each primer, and $2 \mathrm{U}$ of Taq polymerase (Roche Applied Science, Nutley, New Jersey, USA). The PCR primers were as follows: (a) TCR $\beta$, forward, $5^{\prime}$-TCCAGTCTCCAAGACACATAATC- $3^{\prime}$ and reverse, $5^{\prime}$-GACCGAAGTACTGTTCATAATTG-3' and (b) Bcl-2, forward, 5'-GTAGCCATTGCAGCTAGGTG-3' and reverse, $5^{\prime}$-CTTTGTGGAACTGTACGGCCCCAGCATGCG- $3^{\prime}$. The cycling parameters were: (a) TCR $\beta, 94^{\circ} \mathrm{C}$ for 7 minutes $\left(94^{\circ} \mathrm{C}\right.$ for 15 seconds, $60^{\circ} \mathrm{C}$ for $15 \mathrm{sec}$ onds, $72^{\circ} \mathrm{C}$ for 45 seconds) $33 / 72^{\circ} \mathrm{C}$ for 2 minutes and (b) $\mathrm{Bcl}-2,94^{\circ} \mathrm{C}$ for 7 minutes $\left(94^{\circ} \mathrm{C}\right.$ for 15 seconds, $60^{\circ} \mathrm{C}$ for 15 seconds, $72^{\circ} \mathrm{C}$ for 15 seconds) $33 / 72^{\circ} \mathrm{C}$ for 2 minutes. Products were separated on agarose gels and visualized by ethidium bromide (EtBr) staining. 
$A b$ 's and immunofluorescent staining. Staining was performed with the following Ab's as described previously (20): FITC-rat anti-CD4 (PharMingen, San Diego, California, USA) and phycoerythrin-rat (PE) CD8 $\alpha$ (Caltag Laboratories Inc., Burlingame, California, USA) using isotype-matched rat myeloma proteins as controls. Propidium iodide (PI) was added to all stains $(5 \mu \mathrm{g} / \mathrm{ml})$ to exclude dead cells from analysis. Data were collected on 20,000-50,000 cells using a Becton-Dickinson FACScan or FACSCalibur (Becton Dickinson Immunocytometry Systems, Mountain View, California, USA) and analyzed with CELLQuest software (Becton Dickinson Immunocytometry Systems).

Adenosine receptor expression. Adenosine receptor expression was analyzed by RT-PCR with RNA prepared from day- 15 fetal thymocytes according to standard protocols. The PCR primers were: $A 1 R$, forward, $5^{\prime}$-AGGCACTTCGCGATGCTA-3' and reverse, 5'CCTTTTTGTTGAGCTGCTTA-3'; $A 2 a R$, forward, $5^{\prime}$ GGATCAACAGCAACCTGC- $3^{\prime}$ and reverse, $5^{\prime}$-CTTCCTTCTGCAGTGTGGA-3'; A2bR, forward, 5'-CAGCTAGAGACGCAAGAC- $3^{\prime}$ and reverse, $5^{\prime}$-GAGGACAGCAGCTTTTATTC-3'; A3R, forward, 5'-GGTCAAGCTGACAGTCAGAT- $3^{\prime}$ and reverse, $5^{\prime}$-CAAACAAGAAGAGAACCAGAAA- $3^{\prime}$; and $\beta$-actin, forward, $5^{\prime}$-CCTAAGGCCAACCGTGAAAAG- $3^{\prime}$ and reverse, $5^{\prime}$-TCTTCATGGTGCTAGGaGCCA- $3^{\prime}$. The PCR conditions for the $A 1 R, A 2 a R, A 3 R$, and $\beta$-actin were: $95^{\circ} \mathrm{C}$ for 5 minutes, $58^{\circ} \mathrm{C}$ for 1 minute, $72^{\circ} \mathrm{C}$ for 1 minute, once; $95^{\circ} \mathrm{C}$ for 1 minute, $58^{\circ} \mathrm{C}$ for 1 minute, $72^{\circ} \mathrm{C}$ for 1 minute, 40 times. The PCR conditions for the A2bR were identical, except that the annealing temperature was $52^{\circ} \mathrm{C}$. The PCR products were separated on agarose gels and visualized by EtBr staining.

dATP measurements. Single cell suspensions were prepared at $4^{\circ} \mathrm{C}$ from $25-40$ cultured thymic lobes. Aliquots were removed for cell counts and CD4/CD8 staining, and the remaining cells were pelleted by a 20second spin at $16,000 \mathrm{~g}$ in a microcentrifuge. After aspiration of supernatant, the thymocytes were resuspended with vigorous vortexing in $0.5 \mathrm{ml}$ of $60 \%$ methanol and extracted overnight on dry ice. dATP levels were determined by HPLC as previously described (7).

$S A H$ bydrolase enzyme assays. Cultured thymic lobes were rinsed with cold PBS and then flash-frozen in liquid nitrogen and stored at $-80^{\circ} \mathrm{C}$ prior to analysis. $\mathrm{SAH}$ hydrolase enzyme activities were determined by measuring the formation of SAH from homocysteine and radiolabeled adenosine as described previously (7).

\section{Results}

A transgenic TCR $\beta$ chain partially corrects the effects of ADA inbibition. ADA deficiency causes a block in thymocyte development past the point of $\beta$ selection (12) because of the accumulation of ADA substrates derived from apoptotic thymocytes failing this developmental checkpoint. This assertion is supported by the rescue of ADA-deficient FTOCs by the pan-caspase inhibitor,
z-VADfmk, along with normalization of levels of dATP. We reasoned that a TCR $\beta$ chain transgene should also correct the effects of ADA deficiency by preventing thymocyte death by neglect due to failure of $\beta$ selection. As expected, dCF inhibited the development of thymocytes in 2-day FTOCs with control littermates of TCR $\beta$ chain transgenic mice, as shown by both a decrease in the cell yield and in the percentages of $\mathrm{CD} 4^{+} \mathrm{CD}^{+}$thymocytes (Figure 1, a and b). As predicted, a TCR $\beta$ chain transgene rescued thymocyte differentiation in ADA-inhibited cultures. TCR $\beta$ transgenic lobes treated with $5 \mu \mathrm{M} \mathrm{dCF}$ produced 17 -fold more $\mathrm{CD}^{+} \mathrm{CD}^{+}$(double positive [DP]) thymocytes than similarly treated littermate control lobes (Figure 1, b and d). Rescue was only partial, however, because dCF-treated transgenic cultures had just $72 \%$ of the number of thymocytes as transgenic media control cultures (Figure 1, $\mathrm{c}$ and d). These data suggest that ADA substrates derive not only from thymocytes that fail $\beta$ selection but also from those that fail positive and negative selection. The correction observed in transgenic cultures likely resulted from thymocyte expansion and differentiation during a period of delayed accumulation of toxic levels of ADA substrates. Expression of the transgene did not prevent dATP accumulation (data not shown), suggesting that this phenomenon occurs for only a very narrow window in time, perhaps because the cultures are not perfectly synchronized and apoptosis

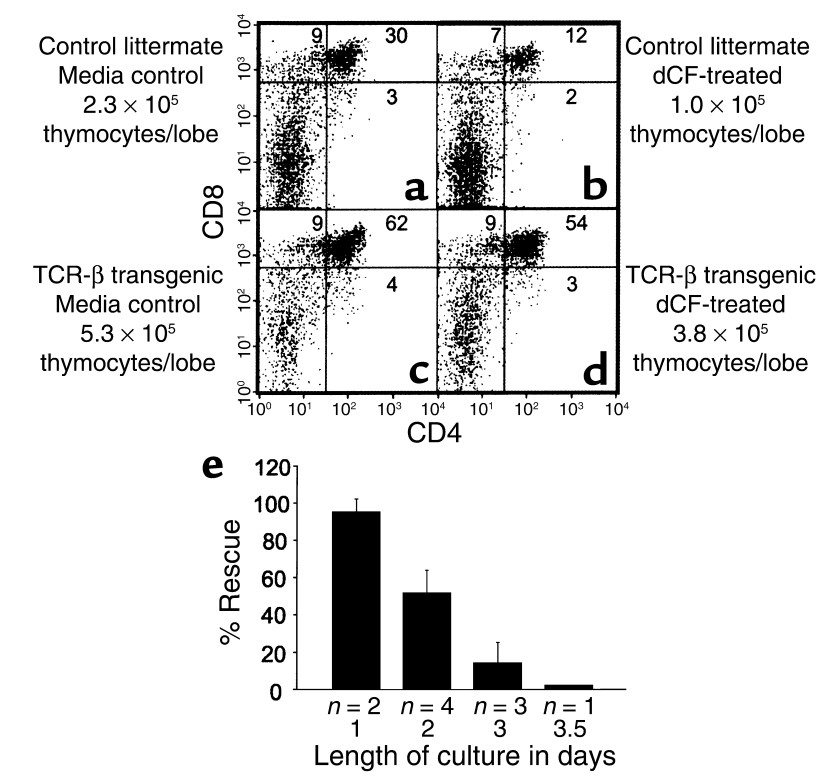

\section{Figure 1}

A transgenic TCR $\beta$ chain partially corrects the effects of ADA inhibition. FTOCs were performed at day 14 of gestation with or without 5 $\mu \mathrm{M} \mathrm{dCF}$ with thymuses from control littermates (a and $\mathbf{b}$ ) and TCR $\beta$ chain transgenic mice (c and $\mathbf{d}$ ). After 2 days, thymocytes were harvested, counted, and stained with FITC-anti-CD4, PE-anti-CD8, and PI to exclude dead cells. (e) The degree of rescue of thymocyte differentiation (mean $\pm S D$ ) is shown as a function of the length of the culture period. The "\% rescue" is defined as (number of DP thymocytes per lobe from dCF-treated transgenic cultures)/(number of DP thymocytes per lobe from control transgenic cultures $) \times 100$. The number of experiments for each time point is shown beneath each bar. 


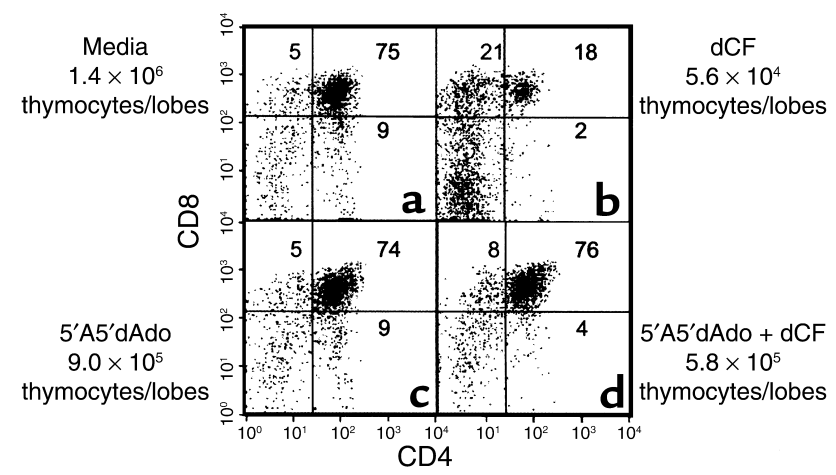

Figure 2

Inhibition of adenosine kinase rescues thymocyte development within ADA-inhibited FTOC. FTOCs were performed with C57BL/ 6 thymuses at day 15 of gestation in media alone (a), $5 \mu \mathrm{M} \mathrm{dCF}(\mathbf{b}), 1 \mu \mathrm{M}$ $5^{\prime} \mathrm{A} 5^{\prime} \mathrm{dAdo}(\mathbf{c})$, or $5 \mu \mathrm{M} \mathrm{dCF}$ plus $1 \mu \mathrm{M} 5^{\prime} \mathrm{A} 5^{\prime} \mathrm{dAdo}(\mathbf{d})$. After 5 days, thymocytes were harvested, counted, and stained with FITC-antiCD4, PE-anti-CD8, and PI to exclude dead cells. The data are representative of one experiment out of four.

due to failure at the positive/negative selection checkpoint may occur in some cells earlier than $\beta$ selection in others. Further experiments revealed that the degree of rescue, defined as (number of DP thymocytes per lobe from dCF-treated transgenic cultures)/(number of DP thymocytes per lobe from control transgenic cultures) $\times 100$, was dependent upon the length of the cultures; rescue was essentially complete in 1-day cultures and declined as the culture period lengthened (Figure 1e). Rescue was also poor if cultures were initiated after a significant number of thymocytes had already passed the $\beta$ selection checkpoint (data not shown).

Inbibition of adenosine kinase corrects thymocyte development within ADA-inbibited FTOC. To differentiate between toxicity induced by nonphosphorylated versus phosphorylated forms of ADA substrates, we evaluated the ability of an adenosine kinase inhibitor to correct thymocyte development in ADA-deficient murine FTOCs. In the mouse, the conversion of adenosine and deoxyadenosine to AMP and dAMP, respectively, is dependent primarily upon the activity of adenosine kinase (21). Treatment of ADA-inhibited lobes with the adenosine kinase inhibitor, $5^{\prime} \mathrm{A} 5^{\prime} \mathrm{dAdo}$, for 5 days resulted in a tenfold increase (range: 5.5 to tenfold) in cell recovery and a 44-fold increase (range: 27 - to 47 -fold) in the absolute numbers of $\mathrm{CD}^{+}{ }^{+} \mathrm{CD}^{+}$thymocytes per lobe (Figure 2, $\mathrm{b}$ and d). Five-day cultures of genetically ADA-deficient fetal thymic lobes $\left(A d a^{m 1 / m 1}\right)$ gave similar results, as did experiments with an alternate adenosine kinase inhibitor, $5^{\prime}$-iodotubercidin (data not shown). These findings demonstrate that inhibition of adenosine kinase largely abrogates the effects of ADA deficiency, as judged by thymocyte differentiation and proliferation, and indicate that the inhibition of thymocyte development in ADA-deficient FTOC is due to a phosphorylated form of an ADA substrate and not adenosine or deoxyadenosine.
Neither aberrant adenosine receptor engagement nor $S A H$ bydrolase inhibition are responsible for the inbibition of thymocyte production in ADA-deficient FTOC. To confirm the assertion that adenosine and deoxyadenosine are not directly responsible for the lymphotoxicity in ADA deficiency, we evaluated two mechanisms by which these molecules have been hypothesized to inhibit lymphocyte development: aberrant engagement of adenosine receptors and inhibition of SAH hydrolase. RT-PCR revealed that $\mathrm{A} 2 \mathrm{aR}, \mathrm{A} 2 \mathrm{bR}$, and $\mathrm{A} 3 \mathrm{R}$ were expressed in murine thymus at day 15 of gestation (Figure 3 ), the time when the FTOC cultures were initiated, validating the potential of these receptors as mediators of toxicity due to ADA inhibition.

To address involvement of individual adenosine receptors in ADA deficiency, fetal thymuses from A2aR and A3R knockout mice were used in ADA-deficient FTOC. The absence of either receptor did not seem to be deleterious to developing thymocytes because CD4/CD8 expression profiles were similar between receptor knockout (Figure 4, c and e) and wild-type fetal thymuses (Figure 4a, and data not shown). An absence of either the $\mathrm{A} 2 \mathrm{aR}$ or $\mathrm{A} 3 \mathrm{R}$ failed to provide protection from the consequences of ADA deficiency. In dCF-treated $A 2 a R^{-/}$FTOCs (Figure 4d) there was a $91 \%$ and greater than $99 \%$ inhibition in the absolute numbers of total and $\mathrm{CD} 4^{+} \mathrm{CD}^{+}$thymocytes per lobe, respectively, compared with control cultures (Figure 4c). Similarly, in FTOCs with $A d a^{m 1 / m 1} A 3 R^{-/-}$thymuses (Figure $4 \mathrm{f}$ ), the yields of total and $\mathrm{CD}^{+}{ }^{+} \mathrm{CD}^{+}$thymocytes were decreased by $96 \%$ and $98 \%$, respectively, compared with FTOCs with $A d a^{+} A 3 R^{-/-}$thymuses (Figure 4e).

We next used the general adenosine receptor agonist, NECA, to evaluate the potential role of the A2bR as well as the possibility that multiple adenosine receptors needed to be engaged to see an effect on thymocyte develop-

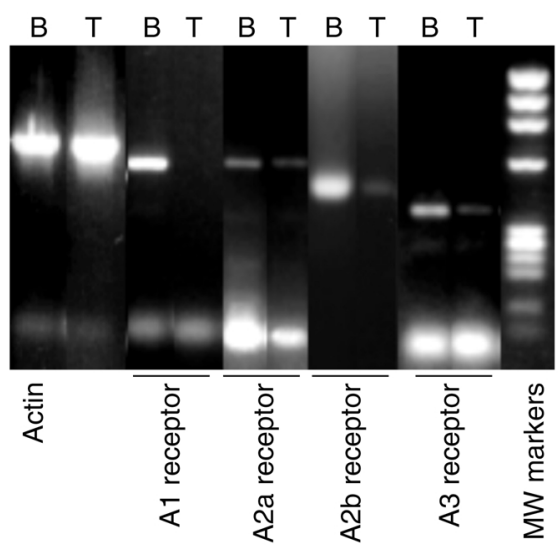

Figure 3

Adenosine receptor expression in murine fetal thymocytes. Adenosine receptor expression was analyzed by RT-PCR with RNA from day-15 fetal thymocytes ( $T$ ). Brain tissue (B) was used as a positive control. RT-PCR was also performed with primers specific for $\beta$-actin. PCR products were visualized by $\mathrm{EtBr}$ staining after separation on a $2 \%$ agarose gel. The molecular-weight markers are a Haelll digest of $\phi \times 174$ DNA. 

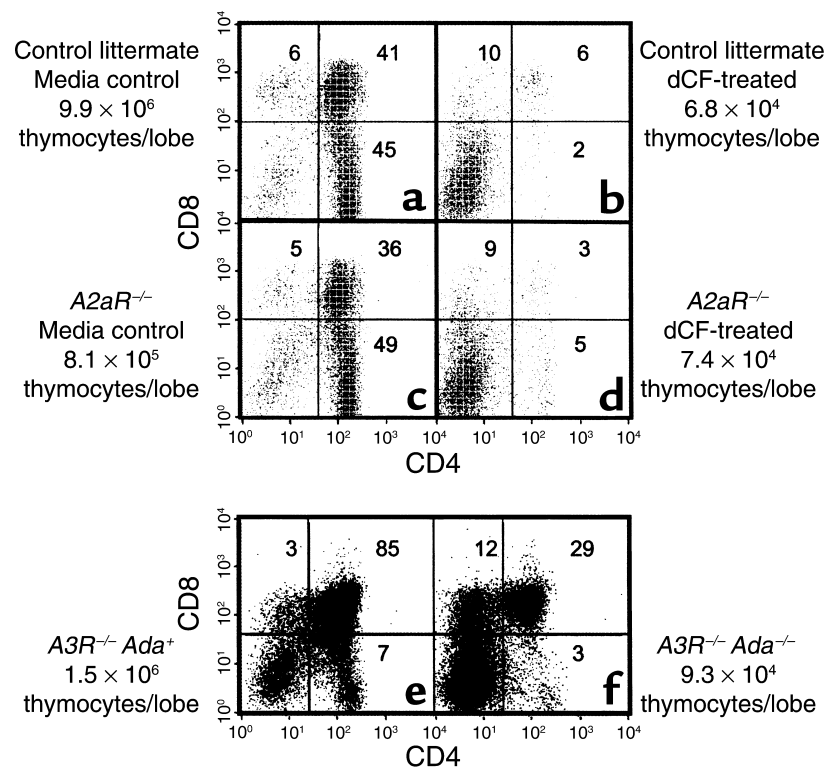

Figure 4

Targeted deletion of the adenosine A2aR or A3R does not correct the effects of ADA deficiency in FTOC. FTOCs were performed at day 15 of gestation with fetal thymuses from $A 2 a R^{-/-}$and $A 3 R^{-/-} A d a^{m 1 / m 1}$ mice and appropriate controls. $A 2 a R^{+/+}(\mathbf{a}$ and $\mathbf{b})$ and $A 2 a R^{-/-}$(c and d) fetal thymic lobes were cultured for 8 days in the presence (b and $\mathbf{d}$ ) or absence (a and $\mathbf{c}$ ) of $5 \mu \mathrm{M} \mathrm{dCF}$. $A 3 R^{-1-} A d a^{+}(\mathbf{e})$ and $A 3 R^{-/-} A d^{m 1 / m 1}$ (f) fetal thymic lobes were cultured for 5 days. At the end of culture, thymocytes were harvested, counted, and stained with FITC-anti-CD4, PE-anti-CD8, and PI to exclude dead cells. The data are representative of one experiment out of two.

ment. However, NECA, at concentrations up to $100 \mu \mathrm{M}$, did not prove deleterious as judged by CD4/CD8 expression profiles and fetal thymic lobe cellularity (Figure 5, $a$ and $b$ ). Likewise, the general adenosine receptor antagonist, XAC, at $25 \mu \mathrm{M}$ failed to protect developing thymocytes from the consequences of ADA deficiency (Figure 5, e and f). FTOCs treated with XAC and dCF exhibited decreases in absolute numbers of total (86\%) and $\mathrm{CD}^{+}{ }^{+} \mathrm{CD}^{+}$thymocytes $(94 \%)$ per lobe, similar to cultures treated with dCF alone ( $85 \%$ and $97 \%$, respectively). Taken together, these data suggest that adenosine receptor engagement is not the mechanism by which ADA deficiency inhibits $T$ cell development.

An additional consequence of ADA deficiency is inhibition of SAH hydrolase by adenosine or deoxyadenosine $(22,23)$, potentially leading to decreased cellular transmethylation reactions (24). In 3-day FTOCs, rescue of ADA-deficient FTOCs by an adenosine kinase inhibitor (Figure 2) did not prevent inhibition of SAH hydrolase (Figure 6); enzyme activity was inhibited by approximately $85 \%$ in ADA-deficient cultures, irrespective of adenosine kinase inhibition. Thus, inhibition of SAH hydrolase is not the mechanism responsible for the inhibition of thymocyte development in ADA-deficient FTOC.

Inbibition of adenosine kinase prevents the accumulation of dATP in ADA-inhibited FTOC. The ability of 5'A5'dAdo to correct thymocyte differentiation in ADA-deficient cul- tures led to the conclusion that lymphotoxicity was caused by a phosphorylated ADA substrate. To confirm this, we measured dATP in ADA-inhibited cultures corrected by $5^{\prime} \mathrm{A} 5^{\prime} \mathrm{dAdo}$. The dATP accumulation was inhibited by $90 \%$ compared with cultures treated with dCF alone (Figure 7). Thus, rescue of thymocyte differentiation in ADA-deficient FTOC by adenosine kinase inhibition correlated with a decrease in dATP levels.

Overexpression of $B c l-2$ corrects the effects of $A D A$ inbibition but does not prevent $d A T P$ accumulation. We reported previously that ADA-deficient FTOCs could be rescued by overexpression of a $\mathrm{Bcl}-2$ transgene (12). To determine whether $\mathrm{Bcl}-2$ acted to prevent death by neglect of thymocytes failing developmental checkpoints (thus limiting the availability of ADA substrates) or to prevent apoptosis induced by ADA substrate accumulation, we measured dATP in dCF-treated FTOCs from $\mathrm{Bcl}-2$ transgenic mice and control littermates (Figure 8). The dATP was elevated an average of 2.5 -fold in the $B c l-2$ cultures compared with those from control littermates. These data indicate that Bcl-2 does not prevent $\mathrm{ADA}$ substrate accumulation, but rather inhibits its effects, and are consistent with mitochondrial-dependent apoptosis as the route by which thymocyte development is impaired by ADA deficiency.

\section{Discussion}

ADA-deficient murine FTOC was used to assess the biochemical mechanism(s) by which a loss of ADA enzyme
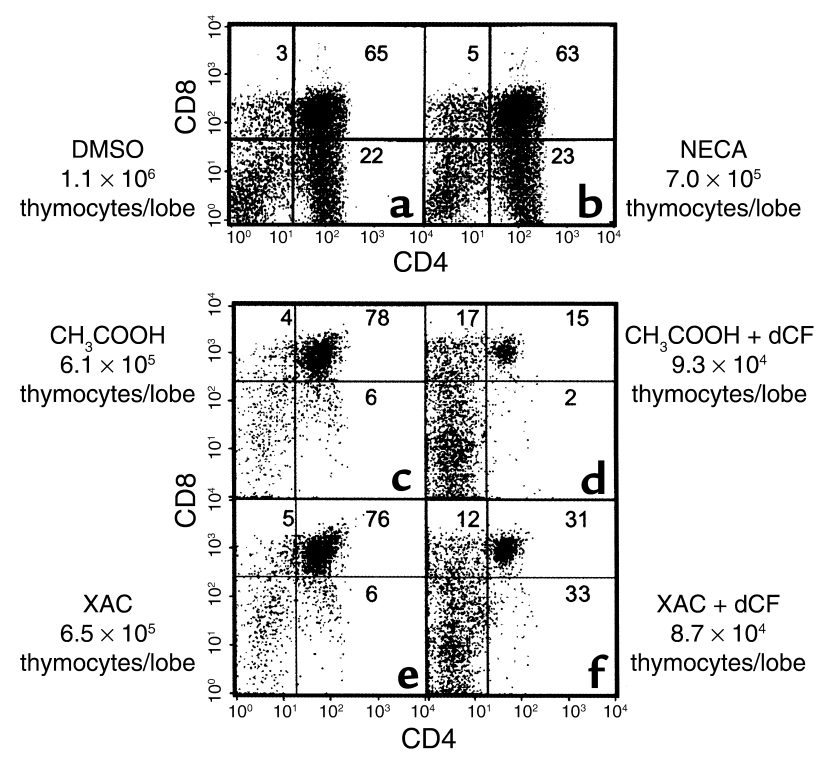

Figure 5

An adenosine receptor agonist does not mimic and an adenosine receptor antagonist does not prevent the effects of ADA deficiency in FTOC. FTOCs were performed with C57BL/ 6 thymuses at day 15 of gestation in the presence of (a) $0.1 \%$ DMSO (carrier for NECA), (b) $100 \mu \mathrm{M}$ $\mathrm{NECA},(\mathbf{c}) 0.1 \mathrm{mM}$ acetic acid (carrier for XAC), (d) $5 \mu \mathrm{M} \mathrm{dCF},(\mathbf{e}) 25$ $\mu \mathrm{MXAC}$, or (f) $25 \mu \mathrm{M}$ XAC plus $5 \mu \mathrm{M} \mathrm{dCF}$. At the end of culture ( 6 days for DMSO and NECA or 2 days for XAC with or without $\mathrm{dCF}$ ), thymocytes were harvested, counted, and stained with FITC-anti-CD4, $\mathrm{PE}-a n t i-\mathrm{CD} 8$, and $\mathrm{PI}$ to exclude dead cells. The data are representative of one experiment out of six for NECA and one out of three for XAC. 


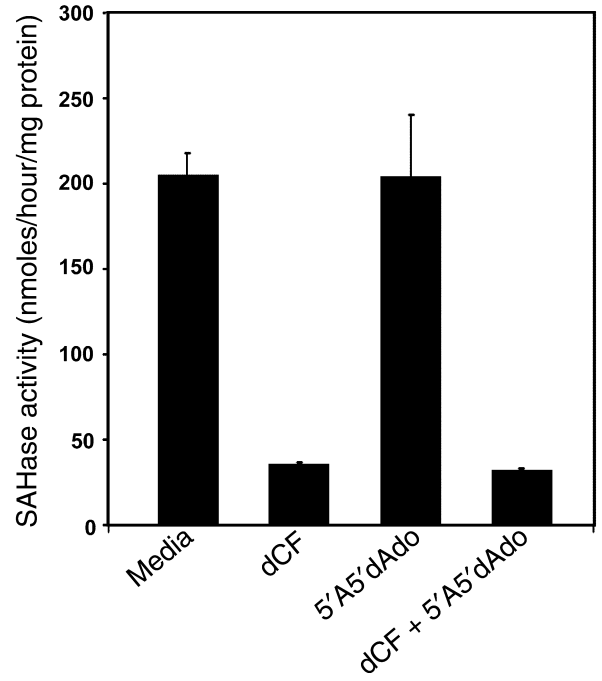

\section{Figure 6}

Rescue of ADA-inhibited FTOCs with an adenosine kinase inhibitor does not prevent inhibition of SAH hydrolase. FTOCs were performed with C57BL/ 6 thymuses at day 15 of gestation with media, $5 \mu \mathrm{M} \mathrm{dCF}, 1 \mu \mathrm{M} 5^{\prime} \mathrm{A} 5^{\prime} \mathrm{dAdo}$, or $5 \mu \mathrm{M} \mathrm{dCF}$ plus $1 \mu \mathrm{M} 5^{\prime} \mathrm{A} 5^{\prime} \mathrm{dAdo}$. After 3 days, SAH hydrolase enzyme activity was assessed in extracts of 25-30 fetal thymic lobes. The data are represented as the means (+ SD) of two independent experiments, each assayed in duplicate.

activity blocks the development of T cells. Previous work from our group (12) demonstrated that the earliest effects of ADA deficiency on thymocyte development were caused by the accumulation of ADA substrates derived from thymocytes undergoing apoptosis. The pan-caspase inhibitor, z-VADfmk, both corrected differentiation and prevented the accumulation of dATP, presumably by inhibiting the death of thymocytes that failed $\beta$ selection. This hypothesis was further corroborated in the present study by the ability of an in-frame $T C R \beta$ chain transgene to partially correct thymocyte differentiation in short-term ADA-deficient FTOCs. The transgene failed to provide substantial protection in cultures longer than 2 days, however, probably because of the accumulation of ADA substrates derived from thymocytes failing positive selection, because a transgenic TCR $\beta$ chain does not assure TCRs competent to recognize self peptide plus MHC when paired with endogenous rearranged $T C R \alpha$ chains. Indeed, dATP was quite elevated in 3.5-day ADA-inhibited FTOCs with $T C R \beta$ chain transgenic thymuses (data not shown).

We next employed 5'A5'dAdo, a potent inhibitor of adenosine kinase, the primary enzyme responsible for the phosphorylation of both adenosine and deoxyadenosine in murine thymus (21), to address the question of whether toxicity is mediated directly by ADA substrates or by phosphorylated derivatives. Correction of thymocyte differentiation with a concomitant $90 \%$ reduction in cellular dATP levels argues strongly against mechanisms of toxicity mediated by adenosine and deoxyadenosine and suggests that the culprit is dATP. To strengthen this assertion, various proposed mechanisms of toxicity due to adenosine and deoxyadenosine or their metabolites were evaluated.

One proposed mechanism of toxicity was adenosine receptor signaling, because engagement of these receptors induces apoptosis in thymocyte suspensions (25-28). However, ADA-deficient FTOCs performed with thymuses from $A 2 a R$ and $A 3 R$ knockout mice revealed no protective effect. Since the absence of a single adenosine receptor might not prevent toxicity, the general adenosine receptor antagonist XAC was tested for its ability to protect ADA-deficient FTOCs. Prevention of adenosine receptor engagement did not affect normal thymocyte differentiation nor did it protect developing thymocytes from the effects of ADA deficiency. In a parallel approach, FTOCs were performed with NECA, a general adenosine receptor agonist, to see if this agent would mimic the consequences of ADA deficiency. NECA, used at concentrations up to 1,000-fold higher than necessary for inducing apoptosis of thymocytes in suspension cultures, had no observable deleterious effects on thymocyte differentiation or proliferation. The reason why NECA induces apoptosis in thymocyte suspensions, but not in FTOC, is unknown, but may be related to protective signals from thymic stromal cells or some other component of the thymic microenvironment. These results suggest that aberrant adenosine receptor engagement is not the mechanism responsible for thymocyte depletion due to ADA deficiency. Our conclusions are thus different from those of Apasov et al. (29) who studied the toxicity of adenosine on thymocyte suspensions in the presence of an ADA inhibitor. However, it is important to note that they used adenosine at $100 \mu \mathrm{M}$, a concentration that is probably never achieved in our FTOCs, and that the consequences of adenosine exposure may be different in isolated thy-

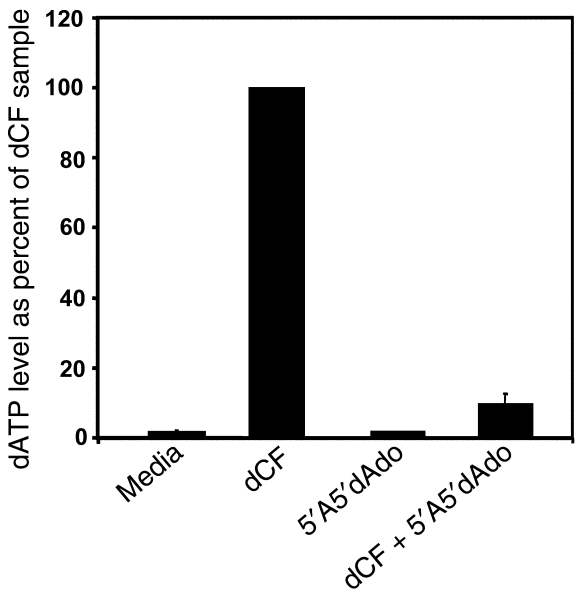

\section{Figure 7}

Inhibition of adenosine kinase prevents the accumulation of dATP in ADA-inhibited FTOC. FTOCs were performed with C57BL/ 6 thymuses at day 15 of gestation with media, $5 \mu \mathrm{M} d C F, 1 \mu \mathrm{M}$ $5^{\prime} \mathrm{A} 5^{\prime} \mathrm{dAdo}$, or $5 \mu \mathrm{M} \mathrm{dCF}$ plus $1 \mu \mathrm{M} 5^{\prime} \mathrm{A} 5^{\prime} \mathrm{dAdo}$. After 2 days, the accumulation of dATP was measured by HPLC in extracts of thymocytes from 30-35 fetal thymic lobes. The dATP levels are expressed as a percentage (mean $+\mathrm{SD}$ ) of those in the dCF-treated thymic lobes from four independent experiments. 


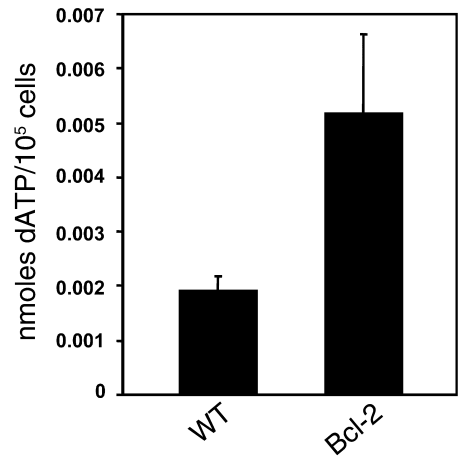

Figure 8

A B Cl-2 transgene does not prevent the accumulation of AATP in ADAinhibited FTOC. FTOCs were performed with $B c l-2$ transgenic mice and wild-type (WT) control littermates at day 15 of gestation with media containing $5 \mu \mathrm{M} \mathrm{dCF}$. After 3 days, the accumulation of dATP was measured by HPLC in extracts of thymocytes from 25-40 fetal thymic lobes of each genotype. The dATP levels are expressed as nanomoles per $10^{5}$ thymocytes (mean \pm SD) from three independent experiments.

mocytes compared with those in the thymic microenvironment as stated above.

Inhibition of SAH hydrolase was another potential mechanism of toxicity mediated by adenosine and deoxyadenosine. This enzyme degrades $\mathrm{SAH}$, a product of transmethylation reactions where S-adenosylmethionine (SAM) is the methyl donor. Elevated adenosine can force reversal of the hydrolytic reaction to form SAH from adenosine and homocysteine (22). Deoxyadenosine acts as a "suicide" inhibitor, forming a covalent bond within the active site of the enzyme (23). Elevated levels of SAH, relative to SAM, as a consequence of SAH hydrolase inhibition, can lead to inhibition of multiple cellular methylation reactions (24). Our observation that SAH hydrolase enzyme activity remained inhibited in ADA-deficient cultures rescued by $5^{\prime} \mathrm{A} 5^{\prime} \mathrm{dAdo}$ argues strongly against $\mathrm{SAH}$ hydrolase inhibition as the mechanism by which ADA deficiency inhibits thymocyte development in FTOC.

With experimental evidence from our lab (12) and others (2) indicating that toxicity associated with ADA deficiency correlated with elevations in dATP, we next explored potential routes of toxicity associated with this molecule. There are two major mechanisms by which dATP could deleteriously affect developing thymocytes: allosteric inhibition of ribonucleotide reductase $(30,31)$, the enzyme that generates deoxyribonucleotides needed for DNA synthesis, and induction of apoptosis (11). Our data indicate that the dATP levels attained in ADA-inhibited FTOCs must be insufficient to inhibit the generation of deoxyribonucleotides, because thymocyte proliferation is normal in dCFtreated FTOCs with $B c l-2$ transgenic thymocytes where dATP levels are hyperelevated.

dATP has the potential to promote apoptosis at two points. First, dATP can induce the release of cytochrome $c$ from isolated mitochondria (11). Cytochrome $c$ release correlates with mitochondrial membrane changes and the release of other proapoptogenic factors, initiating the irreversible effector phase of apoptosis and making this event the "point of no return" in the execution of cell suicide programs $(32,33)$. Second, dATP interacts with Apaf-1, cytochrome $c$, and procaspase- 9 to form the apoptosome (34), resulting in activation of caspase-9 and triggering of the apoptotic cascade. We favor the more proximal point of dATP action because overexpression of $\mathrm{Bcl}-2$, a protein that regulates cytochrome $c$ efflux to the cytoplasm, provides more protection for ADA-deficient FTOCs than deletion of Apaf-1 (12). This would be expected if cytochrome $c$ release were the critical event, given that $\mathrm{Bcl}-2$ is upstream of Apaf- 1 . If the primary role of dATP were to contribute to apoptosome formation, removal of Apaf- 1 or other components of the apoptosome should be equally effective in affording protection to ADA-deficient FTOCs. This is an important distinction, because a deoxyadenosine analogue, 2-chlorodeoxyadenosine, has been proposed to trigger apoptosis of leukemic cells via the interaction of its triphosphorylated derivative, 2-Cl-dATP, with subapoptogenic levels of cytochrome $c$ present in the cytoplasm (35). Further experimentation will be necessary to prove directly that dATP causes cytochrome $c$ release from mitochondria in ADA-deficient FTOCs. Our data do not rule out the possibility that dATP could act indirectly by interfering with some crucial event in thymocyte development such that apoptosis is induced in a mitochondrial-dependent fashion. However, this unspecified event is unlikely to be TCR gene rearrangement, because this was found to be normal in ADA-deficient FTOCs (12).

Our experiments also provide a new perspective on the routes by which thymocytes die by apoptosis during development. The observation that dATP is hyperelevated in ADA-deficient FTOCs with thymuses from $B c l-2$ transgenic mice argues that $\mathrm{Bcl}-2$ does not prevent the death of thymocytes that fail positive/negative selection - otherwise, dATP levels would have normalized. Furthermore, the fact that dATP is hyperelevated relative to dCF-treated FTOCs with wild-type thymuses suggests that larger quantities of ADA substrates are generated from cells failing positive/negative selection than from those failing $\beta$ selection. This is not surprising given the high degree of cellular expansion when thymocytes pass the $\beta$ selection checkpoint (36). Experiments are underway to determine whether thymocytes at later stages of development are also sensitive to the consequences of elevated dATP, and if so, by what mechanism. It will also be important to determine whether our conclusions are applicable to human thymocyte development by using a model system, such as chimeric human/mouse FTOC (37), where human thymocyte development takes place in vitro. These studies are also in progress. We anticipate that continued use of in vitro models of thymocyte differentiation to study ADA deficiency will not only shed additional light on the pathogenesis of this immunodeficiency but will also further our understanding of normal programs of $\mathrm{T}$ cell development. 


\section{Acknowledgments}

We gratefully acknowledge Xiao-Hong Sun and Frank Carbone for gifts of transgenic mice and Marc Parmentier for support of experiments involving A2a-deficient mice. We also thank Kerry Humphrey for manuscript preparation. This work was supported by grants from the NIH (AI-18220 and HD-36044 to L.F. Thompson and AI-43572 to M.R. Blackburn).

1. Giblett, E.R., Anderson, J.E., Cohen, F., Pollara, B., and Meuwissen, H.J. 1972. Adenosine-deaminase deficiency in two patients with severely impaired cellular immunity. Lancet. 2:1067-1069.

2. Hershfield, M.S., and Mitchell, B.S. 1995. Immunodeficiency diseases caused by adenosine deaminase deficiency and purine nucleoside phosphorylase deficiency. In The metabolic and molecular bases of inherited disease. C.R. Scriver A.L. Beaudet, W.S. Sly, and D. Valle, editors. McGraw-Hill Inc. New York, New York, USA. 1725-1768.

3. Surh, C.D., and Sprent, J. 1994. T-cell apoptosis detected in situ during positive and negative selection in the thymus. Nature. 372:100-102.

4. Smith, C.M., and Henderson, J.F. 1982. Deoxyadenosine triphosphate accumulation in erythrocytes of deoxycoformycin-treated mice. Biochem. Pharmacol. 31:1545-1551.

5. Chan, T. 1979. Purine excretion by mouse peritoneal macrophages lacking adenosine deaminase activity. Proc. Natl. Acad. Sci. USA. 76:925-929.

6. Migchielsen, A.A.J., et al. 1995. Adenosine-deaminase-deficient mice die perinatally and exhibit liver-cell degeneration, atelectasis and small intestinal cell death. Nat. Genet. 10:279-287.

7. Wakamiya, M., et al. 1995. Disruption of the adenosine deaminase gene causes hepatocellular impairment and perinatal lethality in mice. Proc. Natl. Acad. Sci. USA. 92:3673-3677.

8. Green, H., and Chan, T. 1973. Pyrimidine starvation induced by adenosine in fibroblasts and lymphoid cells: role of adenosine deaminase. Science. 182:836-837.

9. Mitchell, B.S., Mejias, E., Daddona, P.E., and Kelley, W.N. 1978. Purinogenic immunodeficiency diseases: selective toxicity of deoxyribonucleosides for T cells. Proc. Natl. Acad. Sci. USA. 75:5011-5014.

10. Ullman, B., Gudas, L.J., Cohen, A., and Martin, D.W., Jr. 1978. Deoxyadenosine metabolism and cytotoxicity in cultured mouse T lymphoma cells: a model for immunodeficiency disease. Cell. 14:365-375.

11. Yang, J.C., and Cortopassi, G.A. 1998. dATP causes specific release of cytochrome C from mitochondria. Biochem. Biophys. Res. Comm. 250:454-457.

12. Thompson, L.F., et al. 2000. Metabolites from apoptotic thymocytes inhibit thymopoiesis in adenosine deaminase-deficient fetal thymic organ cultures. J. Clin. Invest. 106:1149-1157.

13. Sentman, C.L., Shutter, J.R., Hockenbery, D., Kanagawa, O., and Korsmeyer, S.J. 1991. bcl-2 inhibits multiple forms of apoptosis but not negative selection in thymocytes. Cell. 67:879-888.

14. Carbone, F.R., Sterry, S.J., Butler, J., Rodda, S., and Moore, M.W. 1992. T cell receptor alpha-chain pairing determines the specificity of residue 262 within the Kb-restricted, ovalbumin $257-264$ determinant. Int. Immunol. 4:861-867.

15. Ledent, C., et al. 1997. Aggressiveness, hypoalgesia and high blood pressure in mice lacking the adenosine $\mathrm{A}_{2 \mathrm{a}}$ receptor. Nature. 388:674-678.

16. Salvatore, C.A., et al. 2000. Disruption of the $\mathrm{A}_{3}$ adenosine receptor gene in mice and its effect on stimulated inflammatory cells. J. Biol. Chem. 275:4429-4434.

17. Agarwal, R.P., Spector, T., and Parks, Jr. R.E. 1977. Tight-binding inhibitors-
IV. Inhibition of adenosine deaminases by various inhibitors. Biochem. Pharmacol. 26:359-367.

18. Miller, R.L., et al. 1979. Adenosine kinase from rabbit liver. II. Substrate and inhibitor specificity. J. Biol. Chem. 254:2346-2352.

19. Boss, G.R., Thompson, L.F., O'Connor, R.D., Ziering, R.W., and Seegmiller, J.E. 1981. Ecto-5' -nucleotidase deficiency: association with adenosine deaminase deficiency and non-association with deoxyadenosine toxicity. Clin. Immunol. Immunopathol. 19:1-7.

20. Fox, R.I., Thompson, L.F., and Huddlestone, J.R. 1981. T cells express T lymphocyte-associated antigens. J. Immunol. 126:2062-2063.

21. Carson, D.A., Kaye, J., and Wasson, D.B. 1980. Differences in deoxyadenosine metabolism in human and mouse lymphocytes. J. Immunol. 124:8-12.

22. Hershfield, M.S. and Kredich, N.M. 1978. S-adenosylhomocysteine hydrolase is an adenosine-binding protein: a target for adenosine toxicity. Science. 202:757-760.

23. Hershfield, M.S. 1979. Apparent suicide inactivation of human lymphoblast S-adenosylhomocysteine hydrolase by 2 -deoxyadenosine and adenine arabinoside. J. Biol. Chem. 254:22-25.

24. Kredich, N.M., and Martin, D.W.,Jr. 1977. Role of S-adenosylhomocysteine in adenosine-mediated toxicity in cultured mouse T lymphoma cells. Cell. 12:931-938.

25. Kizaki, H., Suzuki, K., Tadakuma, T., and Ishimura, Y. 1990. Adenosine receptor-mediated accumulation of cyclic AMP-induced T lymphocyte death through internucleosomal DNA cleavage. J. Biol. Chem. 265:5280-5284.

26. Jondal, M., Okret, S., and McConkey, D. 1993. Killing of immature $\mathrm{CD} 4+\mathrm{CD} 8+$ thymocytes in vivo by anti-CD3 or 5'-(N-ethyl)-carboxamidoadenosine is blocked by glucocorticoid receptor antagonist RU-486. Eur. J. Immunol. 23:1246-1250.

27. McConkey, D. J., Orrenius, S., and Jondal, M. 1990. Agents that elevate cAMP stimulate DNA fragmentation in thymocytes. J. Immunol. 145:1227-1230

28. Resta, R., et al. 1997. Insights into thymic purine metabolism and adenosine deaminase deficiency revealed by transgenic mice overexpressing ecto- 5 nucleotidase (CD73). J. Clin. Invest. 99:676-683.

29. Apasov, S., Chen, J.-F., Smith, P., and Sitkovsky, M. 2000. A $\mathrm{A}_{\mathrm{a}}$ receptor dependent and $\mathrm{A}_{2 \mathrm{a}}$ receptor independent effects of extracellular adenosine on murine thymocytes in conditions of adenosine deaminase deficiency. Blood. 95:3859-3867.

30. Moore, E.C., and Hurlbert, R.B. 1966. Regulation of mammalian deoxyribonucleotide biosynthesis by nucleotides as activators and inhibitors. J. Biol. Chem. 241:4802-4809.

31. Reichard, P. 1972. Control of deoxyribonucleotide synthesis in vitro and in vivo. Adv. Enzyme Regul. 10:3-16.

32. Gottlieb, R.A. 2001. Mitochondria and apoptosis. Biol. Signals Recept. 10:147-161.

33. Penninger,J.M., and Kroemer, G. 1998. Molecular and cellular mechanisms of T lymphocyte apoptosis. Adv. Immunol. 68:51-144.

34. Zou, H., Li, Y., Liu, X., and Wang, X. 1999. An APAF-1.cytochrome $c$ multimeric complex is a functional apoptosome that activates procaspase-9.J. Biol. Chem. 274:11549-11556.

35. Genini, D., et al. 2000. Nucleotide requirements for the in vitro activation of the apoptosis protein-activating factor-1-mediated caspase pathway. J. Biol. Chem. 275:29-34.

36. Dudley, E.C., Petrie, H.T., Shah, L.M., Owen, M., and Hayday, A.C. 1994. T cell receptor beta chain gene rearrangement and selection during thymocyte development in adult mice. Immunity. 1:83-93.

37. Res, P., et al. 1996. CD34+CD38dim cells in the human thymus can differentiate into $T$, natural killer, and dendritic cells but are distinct from pluripotent stem cells. Blood. 87:5196-5206. 